\title{
UPPER BOUNDS FOR THE EIGENVALUES OF DIFFERENTIAL EQUATIONS
}

SAMIR KARAA

Received 10 February 2004; Accepted 4 May 2004

We establish upper bounds for the eigenvalues of second-order and fourth-order differential equations. The inequalities are obtained via rearrangements of higher degree.

Copyright (c) 2006 Hindawi Publishing Corporation. All rights reserved.

\section{Introduction}

Let $L_{+}^{1}(0, l)$ denote the set of all nonnegative functions from $L^{1}(0, l), l$ being a positive real number. The decreasing rearrangement of a function $f \in L_{+}^{1}(0, l)$ is defined by

$$
f^{*}(x)=\sup \left\{t>0: \mu_{f}(t)>x\right\}
$$

for all $x$ in $[0, l]$, where

$$
\mu_{f}(t)=|\{x \in(0, l): f(x)>t\}|, \quad t \geq 0,
$$

is the distribution function of $f$. The increasing rearrangement of $f$ is simply $f^{* *}(x) \equiv$ $f^{*}(l-x)$. The function $f^{*}$ is nonnegative, right continuous and we have (see for instance $[1,8])$

$$
\begin{gathered}
\int_{0}^{x} f d t \leq \int_{0}^{x} f^{*} d t, \quad x \in[0, l], \\
\int_{0}^{l} f d t=\int_{0}^{l} f^{*} d t .
\end{gathered}
$$

Furthermore, if $g \in L^{\infty}(0, l)$ is nonnegative, then we have

$$
\int_{0}^{l} f^{* *} g^{*} d x \leq \int_{0}^{l} f g d x \leq \int_{0}^{l} f^{*} g^{*} d x .
$$

We denote by $f_{n}^{+}$(resp., $f_{n}^{-}$) the symmetrically increasing (resp., decreasing) rearrangement of $f$ of degree $n$. The function $f_{n}^{+}$is uniquely defined by the following conditions 
(see $[11])$ :

(i) $f_{n}^{+}$is periodic on $[0, l / n]$ with period $l / n$,

(ii) $f_{n}^{+}$is symmetric in $[0, l / n]$ about $l /(2 n)$,

(iii) $f_{n}^{+}(x)=f^{*}(2 n x)$ for $x \in[0, l /(2 n)]$.

The function $f_{n}^{-}$is uniquely defined by (i)-(ii) and (iii)': $f_{n}^{-}(x)=f^{* *}(2 n x)$ for $x \in$ $[0, l /(2 n)]$. For more information on rearrangements see $[1,11]$.

In this paper, we derive upper bounds for eigenvalues of some classes of differential equations via rearrangements of higher degree. Many results concerning lower and upper bounds for eigenvalues have been obtained [2-5, 9-11]. For instance in [5], lower bounds for the first eigenvalues of the equations $\left(p(x) y^{\prime}\right)^{\prime}+q(x) y+\mu y=0$ and $\left(p(x) y^{\prime \prime}\right)^{\prime \prime}+$ $q(x) y+\mu y=0$, subject to Dirichlet boundary conditions, are found. In [11], lower and upper bounds for the $n$th eigenvalue of the equation $y^{\prime \prime}+\mu \rho(x) y=0$ are obtained via rearrangements of higher degree. In [7], it is shown that the first eigenvalue of Euler problem decreases when one replaces the design (coefficient function) involved in the problem by its decreasing rearrangement. In [9], we derived upper bounds for the $n$th eigenvalue of the equation $y^{\prime \prime}+p(x) y+\mu q(x) y=0$ with Dirichlet boundary conditions, under some conditions on the coefficients $p$ and $q$. The first goal of this paper is to complete the results obtained in [9], by deriving upper bounds for the eigenvalues of the problem

$$
\left(p(x) y^{\prime}\right)^{\prime}+\lambda q(x) y=0, \quad 0<x<l, y(0)=y(l)=0 .
$$

This will be achieved by first considering the intermediate problem in (2.1). The second goal is to establish upper bounds for fourth-order differential equations. In order to simplify the presentation, we assume throughout the paper that the functions $p$ and $q$ are positive and continuous on $[0, l]$.

\section{Second-order problem}

Consider the boundary value problem

$$
\left(p(x) y^{\prime}\right)^{\prime}+\mu q(x) y=0, \quad y(0)=0, y^{\prime}(l)=0 .
$$

According to a variational principle, the first eigenvalue $\mu_{1}(p, q)$ of this problem can be written as

$$
\mu_{1}(p, q)=\inf _{y \in H^{1}(0, l), y(0)=0} \frac{\int_{0}^{l} p(x) y^{\prime}(x)^{2} d x}{\int_{0}^{l} q(x) y(x)^{2} d x} .
$$

Another characterization of $\mu_{1}(p, q)$ can be found in $[6,7]$

$$
\frac{1}{\mu_{1}(p, q)}=\max _{v \in L^{2},\|v\|=1} \int_{0}^{l} \frac{1}{p(x)}\left(\int_{x}^{l} q(t)^{1 / 2} v(t) d t\right)^{2} d x,
$$

where $\|\cdot\|$ denotes the standard $L^{2}(0, l)$ norm. This maximum is attained at $v_{1}=q^{1 / 2} y_{1}$ where $y_{1}$ is a first eigenfunction of (2.1). Let $\bar{v}=v_{1}$ when $(p, q)=\left(p^{*}, q^{*}\right)$. If $\bar{v}$ is chosen 
nonnegative then the function $x \mapsto \int_{x}^{l} q^{*}(t)^{1 / 2}(t) \bar{v}(t) d t$ is nonincreasing. Thus, arguing as in [7], we have

$$
\begin{aligned}
\frac{1}{\mu_{1}\left(p^{*}, q^{*}\right)} & =\int_{0}^{l} \frac{1}{p^{*}(x)}\left(\int_{x}^{l} q^{*}(t)^{1 / 2} \bar{v}(t) d t\right)^{2} d x \\
& \leq \int_{0}^{l} \frac{1}{p(x)}\left(\int_{x}^{l} q^{*}(t)^{1 / 2} \bar{v}(t) d t\right)^{2} d x \leq \frac{1}{\mu_{1}\left(p, q^{*}\right)}
\end{aligned}
$$

The first inequality follows from the fact that $(1 / p)^{* *}=1 / p^{*}$ and from (1.4). As $\bar{v}$ is not necessarily nondecreasing, we cannot introduce $q(t)$ in these inequalities. We then return to representation (2.2). Let $\bar{y}$ be an eigenfunction of (2.1) associated with $\mu_{1}\left(p, q^{*}\right) \cdot \bar{y}^{2}$ is obviously nondecreasing. Hence, by using (1.4), we have

$$
\mu_{1}\left(p, q^{*}\right)=\frac{\int_{0}^{l} p(x) \bar{y}^{\prime}(x)^{2} d x}{\int_{0}^{l} q^{*}(x) \bar{y}(x)^{2} d x} \geq \frac{\int_{0}^{l} p(x) \bar{y}^{\prime}(x)^{2} d x}{\int_{0}^{l} q(x) \bar{y}(x)^{2} d x} \geq \mu_{1}(p, q) .
$$

If $\mu_{1}\left(p, q^{*}\right)=\mu_{1}(p, q)$ then $\bar{y}$ is also an eigenfunction for $\mu_{1}(p, q)$ and therefore $q^{*}=q$. The equality $\mu_{1}\left(p^{*}, q^{*}\right)=\mu_{1}\left(p, q^{*}\right)$ leads similarly to $p=p^{*}$. We have then

Theorem 2.1. $\mu_{1}(p, q) \leq \mu_{1}\left(p^{*}, q^{*}\right)$ and equality holds only if $(p, q)=\left(p^{*}, q^{*}\right)$.

Now let $\nu_{2}(p, q)$ denote the second eigenvalue of the following problem

$$
\left(p(x) y^{\prime}\right)^{\prime}+\lambda q(x) y=0, \quad y^{\prime}(0)=0, y^{\prime}(l)=0,
$$

and let $a$ be the unique zero in $(0, l)$ of the eigenfunction associated with $v_{2}(p, q)$. Then by applying Theorem 2.1 on each subinterval $[0, a]$ and $[a, l]$ and by arguing as in the proof of $[9$, Theorem 7.4$]$, we can show that $\nu_{2}(p, q) \leq \nu_{2}\left(p_{1}^{-}, q_{1}^{-}\right)$. More generally, we have the following result.

Theorem 2.2. Let $v_{n}(p, q)$ be the nth eigenvalue of problem (2.6). Then, $v_{n}(p, q) \leq v_{2}\left(p_{n-1}^{-}\right.$, $\left.q_{n-1}^{-}\right)$for all $n>1$, and equality holds only if $(p, q)=\left(p_{n-1}^{-}, q_{n-1}^{-}\right)$.

The next result is a direct consequence of this theorem and arguments from [9].

Theorem 2.3. Let $\lambda_{n}(p, q)$ be the nth eigenvalue of

$$
\left(p(x) y^{\prime}\right)^{\prime}+\lambda q(x) y=0, \quad y(0)=0, y(l)=0 .
$$

Then, $\lambda_{n}(p, q) \leq \lambda_{n}\left(p_{n}^{+}, q_{n}^{+}\right)$for all $n$, and equality holds only if $(p, q)=\left(p_{n}^{+}, q_{n}^{+}\right)$.

\section{Fourth-order problem}

Let $\mu_{1}(p, q)$ denote the first eigenvalue of the fourth-order differential equation

$$
\begin{gathered}
\left(p(x) y^{\prime \prime}\right)^{\prime \prime}+\mu q(x) y=0 \\
y(0)=y^{\prime \prime}(0)=0, \quad y^{\prime}(l)=y^{\prime \prime \prime}(l)=0 .
\end{gathered}
$$


Let $\psi=p y^{\prime \prime}$. Then $\psi^{\prime \prime}=-\mu q(x) y$ and $\psi(0)=\psi^{\prime}(l)=0$. It follows that

$$
\psi(x)=\mu \int_{0}^{l} g(x, t) q(t) y(t) d t,
$$

where $g(x, t)=\min \{x, t\}$. Hence,

$$
y^{\prime \prime}(x)=\frac{\mu}{p(x)} \int_{0}^{l} g(x, t) q(t) y(t) d t .
$$

Since the right-hand member belongs to $L^{1}(0, l)$ and $y(l)=y^{\prime}(l)=0$, we have

$$
y(x)=\mu \int_{0}^{l} g(x, t) \frac{1}{p(t)}\left(\int_{0}^{l} g(t, s) q(s) y(s) d s\right) d t .
$$

Let $L$ be the operator defined by

$$
(L v)(x)=\int_{0}^{l} g(x, t) q(x)^{1 / 2} p(t)^{-1 / 2} v(t) d t
$$

$v \in L^{2}(0, l)$. The kernel $l(x, t)=g(x, t) q(x)^{1 / 2} p(t)^{-1 / 2}$ is square integrable and therefore $L$ is a Hilbert-Schmidt operator from $L^{2}(0, l)$ in $L^{2}(0, l)$. It is in particular compact on $L^{2}(0, l)$. Its adjoint $L^{*}$ is given by

$$
\left(L^{*} v\right)(x)=\int_{0}^{l} g(x, t) q(t)^{1 / 2} p(x)^{-1 / 2} v(t) d t,
$$

$v \in L^{2}(0, l)$.Equation (3.4) can be written now as

$$
v=\mu\left(L L^{*}\right) v,
$$

where $v(x)=q(x)^{1 / 2} y(x)$. The operator $\left(L L^{*}\right)$ is compact, selfadjoint and positive, therefore there exists an infinite sequence of eigenvalues $0<\mu_{1}(p, q) \leq \mu_{2}(p, q) \leq \cdots$ which increases without limit. The first eigenvalue can be found as

$$
\frac{1}{\mu_{1}}=\max _{v \in L^{2} \backslash\{0\}} \frac{\left\|L^{*} v\right\|^{2}}{\|v\|^{2}} .
$$

The maximum is attained at $v_{1}=q^{1 / 2} y_{1}$, where $y_{1}$ is the first eigenfunction of (3.1). From general theory of compact operators with positive kernels [6], we know that $\mu_{1}$ is simple and $y_{1}$ can be chosen positive on $[0, l]$. We finally notice that

$$
\int_{0}^{l} g(x, t) q(t)^{1 / 2} v(t) d t=\int_{0}^{x}\left(\int_{t}^{l} q(s)^{1 / 2} v(s) d s\right) d t
$$

and therefore $\mu_{1}$ can be found as

$$
\frac{1}{\mu_{1}}=\max _{\|v\|=1} \int_{0}^{l} \frac{1}{p(x)}\left(\int_{0}^{x} \int_{t}^{l} q(s)^{1 / 2} v(s) d s d t\right)^{2} d x .
$$


Let $\bar{v}=\left(q^{*}\right)^{1 / 2} \bar{y}$, where $\bar{y}$ is a positive eigenfunction corresponding to $\mu_{1}\left(p^{* *}, q^{*}\right)$ and such that $\|\bar{v}\|=1$. We have

$$
\begin{aligned}
\frac{1}{\mu_{1}\left(p^{* *}, q^{*}\right)} & =\int_{0}^{l}\left(\frac{1}{p}\right)^{*}\left(\int_{0}^{x} \int_{t}^{l} q^{*}(s)^{1 / 2} \bar{v}(s) d s d t\right)^{2} d x \\
& \leq \int_{0}^{l} \frac{1}{p(x)}\left(\int_{0}^{x} \int_{t}^{l} q^{*}(s)^{1 / 2} \bar{v}(s) d s d t\right)^{2} d x \leq \frac{1}{\mu_{1}\left(p, q^{*}\right)}
\end{aligned}
$$

On the other hand, reasoning as in the previous section, we find that $\mu_{1}\left(p, q^{*}\right) \geq \mu_{1}(p, q)$. As a result, we have

Theorem 3.1. $\mu_{1}(p, q) \leq \mu_{1}\left(p^{* *}, q^{*}\right)$ and equality holds only if $(p, q)=\left(p^{* *}, q^{*}\right)$.

By using this theorem and arguments from [9], we prove the following.

Theorem 3.2. Let $\lambda_{n}(p, q)$ be the nth eigenvalue of

$$
\begin{gathered}
\left(p(x) y^{\prime \prime}\right)^{\prime \prime}-\lambda q(x) y=0, \\
y(0)=y^{\prime \prime}(0)=0, \quad y(l)=y^{\prime \prime}(l)=0 .
\end{gathered}
$$

Then, $\lambda_{n}(p, q) \leq \lambda_{n}\left(p_{n}^{-}, q_{n}^{+}\right)$for all $n$, and equality holds only if $(p, q)=\left(p_{n}^{-}, q_{n}^{+}\right)$.

\section{Another problem}

In this section, we denote by $\mu_{1}(p, q)$ the first eigenvalue of the following problem

$$
\begin{gathered}
y^{(4)}-[\mu q(x)-p(x)] y=0, \\
y(0)=y^{\prime \prime}(0)=0, \quad y^{\prime}(l)=y^{\prime \prime \prime}(l)=0 .
\end{gathered}
$$

Let $h$ and $H$ be positive numbers such that $h \leq q(x) \leq H$ on $[0, l]$. Then, we have

LEMMA 4.1. If

$$
H \max _{x} p(x)-h \min _{x} p(x) \leq h \pi^{4} l^{-4} / 16
$$

then $p(x)-\mu_{1}(p, q) q(x) \leq 0$ for all $x \in[0, l]$. If $y_{1}(x)$ is a positive first eigenfunction of problem (4.1) and if condition (4.2) holds, then $y_{1}(x)$ is nondecreasing.

Proof. We have

$$
\begin{aligned}
\mu_{1}(p, q) & \geq \inf _{y}\left[\int_{0}^{l} y^{\prime \prime 2} d x / \int_{0}^{l} q y^{2} d x\right]+\min _{x} p(x) / H \\
& \geq H^{-1}[\pi /(2 l)]^{4}+\min _{x} p(x) / H .
\end{aligned}
$$

If (4.2) holds, then from the last inequality we get $\mu_{1}(p, q) \geq \max _{x} p(x) / h$, and therefore $\mu_{1}(p, q) q(x)-p(x) \geq 0$ for all $x \in[0, l]$. If $y_{1}(x)$ is a positive eigenfunction of problem (4.1) associated with $\mu_{1}(p, q)$, then $y_{1}^{(4)}(x)=\left[\mu_{1}(p, q) q(x)-p(x)\right] y_{1}(x) \geq 0$ in $[0, l]$. This means that $y_{1}^{\prime \prime}(x)$ is a convex function. As $y_{1}^{\prime \prime}(0)=y_{1}^{\prime \prime \prime}(l)=0$, it follows that $y_{1}^{\prime \prime}(x)$ is 
nonnegative and therefore $y_{1}(x)$ is concave and nondecreasing, since $y_{1}(0)=y_{1}^{\prime}(l)=0$.

Theorem 4.2. If condition (4.2) holds, then

$$
\mu_{1}(p, q) \leq \mu_{1}\left(p^{* *}, q^{*}\right) .
$$

Moreover, equality holds only if $(p, q)=\left(p^{* *}, q^{*}\right)$.

Proof. Suppose that (4.2) is fulfilled and let $y_{1}(x)$ be a positive first eigenfunction of problem (4.1) associated with the couple $\left(p^{* *}, q^{*}\right)$. Since $p^{* *}$ and $q^{*}$ satisfy also condition (4.2), Lemma 4.1 tells us that $y_{1}(x)$ in nondecreasing. Therefore, we have

$$
\mu_{1}\left(p^{* *}, q^{*}\right) \geq \frac{\int_{0}^{l} y_{1}^{\prime \prime 2} d x+\int_{0}^{l} p y_{1}^{2} d x}{\int_{0}^{l} q y_{1}^{2} d x} \geq \mu_{1}(p, q),
$$

and it is easily seen that equality holds only when $(p, q)=\left(p^{* *}, q^{*}\right)$.

Once again, by using Theorem 4.2 and arguments from [9], we prove

Theorem 4.3. Let $\lambda_{n}(p, q)$ be the nth eigenvalue of

$$
\begin{gathered}
y^{(4)}-[\lambda q(x)-p(x)] y=0, \\
y(0)=y^{\prime \prime}(0)=0, \quad y(l)=y^{\prime \prime}(l)=0 .
\end{gathered}
$$

Then, $\lambda_{n}(p, q) \leq \lambda_{n}\left(p_{n}^{-}, q_{n}^{+}\right)$for all $n$, and equality holds only if $(p, q)=\left(p_{n}^{-}, q_{n}^{+}\right)$.

We have in particular the following.

Corollary 4.4. Let $\lambda_{n}(p)$ denote the first eigenvalue of the problem

$$
\begin{gathered}
y^{(4)}-[\lambda-p(x)] y=0, \\
y(0)=y^{\prime \prime}(0)=0, \quad y(l)=y^{\prime \prime}(l)=0 .
\end{gathered}
$$

If $\max _{x} p(x)-\min _{x} p(x) \leq \pi^{4} /(2 l)^{4}$, then

$$
\lambda_{n}(p) \leq \lambda_{n}\left(p_{n}^{-}\right)
$$

Moreover, equality holds only if $p=p_{n}^{-}$.

\section{References}

[1] C. Bandle, Isoperimetric Inequalities and Applications, Monographs and Studies in Mathematics, vol. 7, Pitman, Massachusetts, 1980.

[2] __ Extremal problems for eigenvalues of the Sturm-Liouville type, General Inequalities, 5 (Oberwolfach, 1986), Internat. Schriftenreihe Numer. Math., vol. 80, Birkhäuser, Basel, 1987, pp. 319-336.

[3] D. O. Banks, Bounds for the eigenvalues of nonhomogeneous hinged vibrating rods, Journal of Mathematics Mechanics 16 (1967), 949-966.

[4] D. C. Barnes, Rearrangements of functions and lower bounds for eigenvalues of differential equations, Applicable Analysis. An International Journal 13 (1982), no. 4, 237-248. 
[5] __ Extremal problems for eigenvalues with applications to buckling, vibration and sloshing, SIAM Journal on Mathematical Analysis 16 (1985), no. 2, 341-357.

[6] J. A. Cochran, The Analysis of Linear Integral Equations, McGraw-Hill, New York, 1972.

[7] S. J. Cox and C. M. McCarthy, The shape of the tallest column, SIAM Journal on Mathematical Analysis 29 (1998), no. 3, 547-554.

[8] G. H. Hardy, J. E. Littlewood, and G. Polya, Inequalities, Cambridge University Press, Cambridge, 1934.

[9] S. Karaa, Sharp estimates for the eigenvalues of some differential equations, SIAM Journal on Mathematical Analysis 29 (1998), no. 5, 1279-1300.

[10] _ Inequalities for eigenvalue functionals, Journal of Inequalities and Applications 4 (1999), no. 2, 175-181.

[11] B. Schwarz, On the extrema of the frequencies of nonhomogeneous strings with equimeasurable density, Journal of Mathematics Mechanics 10 (1961), 401-422.

Samir Karaa: Department of Mathematics and Statistics, Sultan Qaboos University, P.O. Box 36, Al-Khod, Muscat 123, Sultanate of Oman

E-mail address: skaraa@squ.edu.om 